Article

\title{
Tumor Regression in Lymph Node Metastases of Esophageal Adenocarcinomas after Neoadjuvant Therapy
}

\author{
Marek Osecky ${ }^{1}$ (D) Dino Kröll ${ }^{2,3}$, Marcus Feith ${ }^{4}$, Daniel Reim ${ }^{4}$ (), Bastian Dislich ${ }^{1}$, \\ Karen Becker ${ }^{5}$ and Rupert Langer $1,6, *$ (D) \\ 1 Institute of Pathology, University of Bern, 3008 Bern, Switzerland; marek.osecky@students.unibe.ch (M.O.); \\ bastian.dislich@pathology.unibe.ch (B.D.) \\ 2 Department of Visceral Surgery and Medicine, Inselspital, Bern University Hospital, University of Bern, \\ 3008 Bern, Switzerland; dino.kroell@charite.de \\ 3 Department of Surgery, Campus Charité Mitte|Campus Virchow-Klinikum, Charité-Universitätsmedizin \\ Berlin, 10117 Berlin, Germany \\ 4 Department of Surgery, Klinikum rechts der Isar, TUM School of Medicine, 81675 Munich, Germany; \\ marcus.feith@tum.de (M.F.); daniel.reim@tum.de (D.R.) \\ 5 Institute of Pathology, Technische Universität München, 81675 Munich, Germany; karen.becker@tum.de \\ 6 Institute of Pathology and Molecular Pathology, Kepler University Hospital and Johannes Kepler University \\ Linz, 4021 Linz, Austria \\ * Correspondence: rupert.langer@kepleruniklinikum.at
}

Received: 11 September 2020; Accepted: 6 October 2020; Published: 16 October 2020

\begin{abstract}
Tumor regression following neoadjuvant treatment can be observed in lymph node (LN) metastases similar to the primary tumor in esophageal adenocarcinomas (EAC). We evaluated the prognostic significance of tumor regression in LN metastases of locally advanced EAC of 239 patients treated with neoadjuvant radiochemotherapy (RCTX) or chemotherapy (CTX) followed by esophagectomy. We examined retrospectively the LN for histopathologic signs of regression, i.e., nodular fibrosis and acellular mucin. LN classification was performed according to two parameters: presence (-) or absence $(+)$ of residual tumor and regression characteristics in the $\mathrm{LN}$, resulting in four categories: $\mathrm{LN}-/ \mathrm{REG}-, \mathrm{LN}-/ \mathrm{REG}+, \mathrm{LN}+/ \mathrm{REG}+, \mathrm{LN}+/ \mathrm{REG}-$. In total, $\mathrm{LN}$ metastases with residual tumor were detectable in 117/239 (49\%) cases. Regression in LN were observed in 85/239 cases (35.5\%). The distribution of the LN/REG categories were as follows: 97 patients (40.6\%) were LN-/REG-. A total of 25 patients (10.5\%) were LN-/REG+. A total of 60 (25.1\%) were LN+/REG+ and $57(23.8 \%)$ $\mathrm{LN}+/$ REG-. The LN/Reg categorization had a significant prognostic value in univariate analysis $(p<0.001)$ and multivariate analysis $(\mathrm{HR}=1.326 ; p=0.002)$ with similar results for the subgroups of patients treated with RCTX or CTX. The prognosis of LN-/REG+ was worse than LN-/REG- but better than both LN+ categories, which was demonstrated in the Kaplan-Meier curves but did not reach statistical significance ( $p=0.104$ and $p=0.090$, respectively). In contrast, there was no difference between $\mathrm{LN}+/ \mathrm{REG}+$ and LN+/REG- $(p=0.802)$. In summary, regression in LN metastases of EAC can be observed in a significant number of patients after neoadjuvant therapy. Complete regression of former LN metastases in comparison to "true" negative LN seems to be of prognostic relevance but additional studies are needed to confirm this trend seen in our study.
\end{abstract}

Keywords: esophagus; adenocarcinoma; regression; lymph node; metastases

\section{Introduction}

Neoadjuvant chemotherapy (CTX) or radiochemotherapy (RCTX) followed by surgery is standard care for locally advanced esophageal adenocarcinomas, as previous studies have provided evidence 
for survival benefit for patients compared to surgery alone [1-6], and both therapy options have been shown to be comparable in the improvement of patient's outcome [7-10]. The morphologic assessment of treatment response is usually performed at the primary tumor site and the degree of regression correlates with long term outcome of the patients [11,12]. Another, if not the most important prognostic factor for esophageal adenocarcinomas (EAC) after neoadjuvant treatment and surgery, is the presence of lymph node metastases (LNM) [13-18]. Tumor regression can be observed in LNM similarly to the primary tumors. Several studies using different, partly rather complex approaches to characterize regressive changes in LN and LNM of esophageal carcinomas have also shown an association between regression of LNM and outcome [19-21].

For gastric cancer, a proposal for a simple categorization of LNM with or without regression has been published [22]. Using a slightly modified categorization system for LN and LNM combining evidence of residual tumor with signs of regression, we recently demonstrated a prognostic impact of regression in LNM in gastric carcinomas (GC), in particular for cases with residual tumor [14].

We now tested whether this categorization system also can be applied in EAC, using well characterized case collections from two surgical centers, where different neoadjuvant therapy procedures (i.e., chemotherapy or radiochemotherapy) were also administered before surgery.

\section{Results}

\subsection{Pathologic Data}

According to the TMN classification, $43 / 239$ primary tumors $(18 \%)$ were described as ypT0, $29(12.1 \%)$ as ypT1, $47(19.7 \%)$ as ypT2, $116(48.5 \%)$ as ypT3 and $4(1.7 \%)$ as ypT4. One hundred twenty-two $(51 \%)$ of the 239 patients showed tumor free lymph nodes after neoadjuvant therapy (ypN0), while 117 (49\%) had LNM with 50 (20.9\%) of them categorized as ypN1, $38(15.9 \%)$ ypN2 and $29(12.1 \%)$ ypN3. Distant metastases were present in 24 patients (10\%). Complete tumor resection (R0) was achieved in 201/239 cases (81.4\%). The tumor regression of the primary tumor according to the tumor regression grade (TRG) system by Becker was classified as TRG1a in 43/239 cases (18\%), TRG1b in 78 cases (32.6\%), TRG2 in 51 cases (21.3\%) and TRG3 in 67 cases (28\%; Table 1$)$.

Table 1. Case cohorts and pathological parameters.

\begin{tabular}{cccccccc}
\hline & \multicolumn{2}{c}{ Total } & \multicolumn{5}{c}{ Cohorts } \\
\hline \multirow{2}{*}{ Parameter } & & & & \multicolumn{2}{c}{ Bern } & Munich \\
\hline \multirow{3}{*}{ ypT category } & ypT0 & 43 & 18 & 36 & 24.5 & 7 & 7.6 \\
& ypT1 & 29 & 12.1 & 20 & 13.6 & 9 & 9.8 \\
& ypT2 & 47 & 19.7 & 28 & 19 & 19 & 20.7 \\
& ypT3 & 116 & 48.5 & 59 & 40.1 & 57 & 61.9 \\
& ypT4 & 4 & 1.7 & 4 & 20.7 & 0 & 0 \\
\hline \multirow{2}{*}{ ypN category } & ypN0 & 122 & 51 & 87 & 59.2 & 35 & 38 \\
& ypN1 & 50 & 20.9 & 31 & 21.1 & 19 & 20.7 \\
& ypN2 & 38 & 15.9 & 20 & 13.6 & 18 & 19.6 \\
& ypN3 & 29 & 12.1 & 9 & 6.1 & 20 & 21.7 \\
\hline \multirow{2}{*}{ distant Metastases } & M0 & 215 & 90 & 136 & 92.5 & 79 & 85.9 \\
& M1 & 24 & 10 & 11 & 7.5 & 13 & 14.1 \\
\hline \multirow{2}{*}{ resection status } & R0 & 201 & 84.1 & 138 & 93.9 & 63 & 68.5 \\
& R1/2 & 38 & 15.9 & 9 & 6.1 & 29 & 31.5 \\
\hline \multirow{2}{*}{ neoadjuvant therapy } & CTX & 114 & 47.7 & 22 & 15 & 92 & 100 \\
& RCTX & 125 & 52.3 & 125 & 85 & 0 & 0 \\
\hline
\end{tabular}


Table 1. Cont.

\begin{tabular}{cccccccc}
\hline & \multicolumn{2}{c}{ Total } & \multicolumn{4}{c}{ Cohorts } \\
\hline \multirow{2}{*}{ Parameter } & & & & \multicolumn{2}{c}{ Bern } & \multicolumn{2}{c}{ Munich } \\
\hline & & N & $\%$ & N & $\%$ & N & $\%$ \\
\hline \multirow{3}{*}{ TRG (Becker) primary tumor } & $1 \mathrm{a}$ & 43 & 18 & 36 & 24.5 & 7 & 7.6 \\
& 2 & 78 & 32 & 56 & 38.1 & 22 & 23.9 \\
& 3 & 51 & 21.3 & 25 & 17 & 26 & 28.3 \\
& & 67 & 28 & 30 & 20.4 & 37 & 40.2 \\
\hline \multirow{2}{*}{ LN category } & LN-/REG- & 97 & 40.6 & 69 & 46.9 & 28 & 30.4 \\
& LN-/REG+ & 25 & 10.5 & 18 & 12.2 & 7 & 7.6 \\
& LN+/REG+ & 60 & 25.1 & 36 & 24.5 & 24 & 26.1 \\
& LN+/REG- & 57 & 23.8 & 24 & 16.3 & 33 & 35.9 \\
\hline
\end{tabular}

\subsection{Regressive Changes in Lymph Nodes}

In total, vital LNM (ypN1-3) were found in 117/239 (49\%) cases after neoadjuvant therapy. The number of resected and examined LN ranged from 5 to 86 (median $=25)$. Regressive changes in LN were observed in 85/239 cases (35.5\%). Using the proposed categorization, 97 patients $(40.6 \%)$ had negative LN without regression (LN-/REG-). A total of 25 (10.5\%) had completely regressed LNM (LN-/REG+), 60 (25.1\%) persistent LNM with regression (LN+/REG+) and 57 (23.8\%) had LNM without evidence of regression (LN+/REG-; Table 1).

\subsection{Correlation with Pathological Data}

A major response of the primary tumor correlated with the categorization of LN/LNM described above. 19/25 of the cases (76\%) with complete LN regression (LN-/REG + ) could also be graduated with TRG1a or TRG1b with decreasing prevalence for LN+/REG+ $(21 / 60 ; 35 \%)$ and for LN+/REG(11/60; 19.3\%). However, some cases also showed different responses in LNM and primary tumor. A total of $6 / 25$ patients $(24 \%)$ with completely regressed LNM showed a poor primary tumor response (TRG2 or TRG3). A total of 43 of 85 cases with presence of LN regression (50.6\%), 4 of them with complete LN regression, showed TRG2 or TRG3. Among the LN+/REG- category, however, no case with complete regression of the primary tumor (TRG1a) was observed (Table 2).

Table 2. Lymph node (LN) categories and Tumor Regression Grades of the primary tumors.

\begin{tabular}{ccccccc}
\hline & & \multicolumn{3}{c}{ TRG (Becker) } & Total \\
& & $\mathbf{1 a}$ & $\mathbf{1 b}$ & $\mathbf{2}$ & $\mathbf{3}$ & \\
\hline \multirow{4}{*}{ LN category } & LN-/REG- & 32 & 38 & 17 & 10 & 97 \\
& LN-/REG+ & 7 & 12 & 2 & 4 & 25 \\
& LN+/REG+ & 4 & 17 & 16 & 23 & 60 \\
& LN+/REG- & 0 & 11 & 16 & 30 & 57 \\
\hline total & & 43 & 78 & 51 & 67 & 239 \\
\hline
\end{tabular}

\subsection{Survival Analysis}

Survival data were available for 226/239 patients. With regard to overall survival, the LN/REG categorization was a prognostically significant parameter in univariate analysis. Patients with negative nodal status after neoadjuvant therapy, regardless of regression status (i.e., LN-/REG- or LN-/REG+), had significantly better survival $(p<0.001)$ than patients with $\mathrm{LN}+/ \mathrm{REG}+$ and $\mathrm{LN}+/ \mathrm{REG}-$ combined. Between the categories LN-/REG- and LN-/REG+, which are summarized as ypN0 in the TNM classification, a trend for a better survival for the former was observed, but without statistical significance $(p=0.104)$. The LN-/REG+ group, which comprised only 25 patients, however, also showed a trend 
for a better survival compared to LN+ patients, regardless of regression $(p=0.090)$. No difference was found between LN+/REG+ and LN+/REG- ( $p=0.802$; Figure 1).

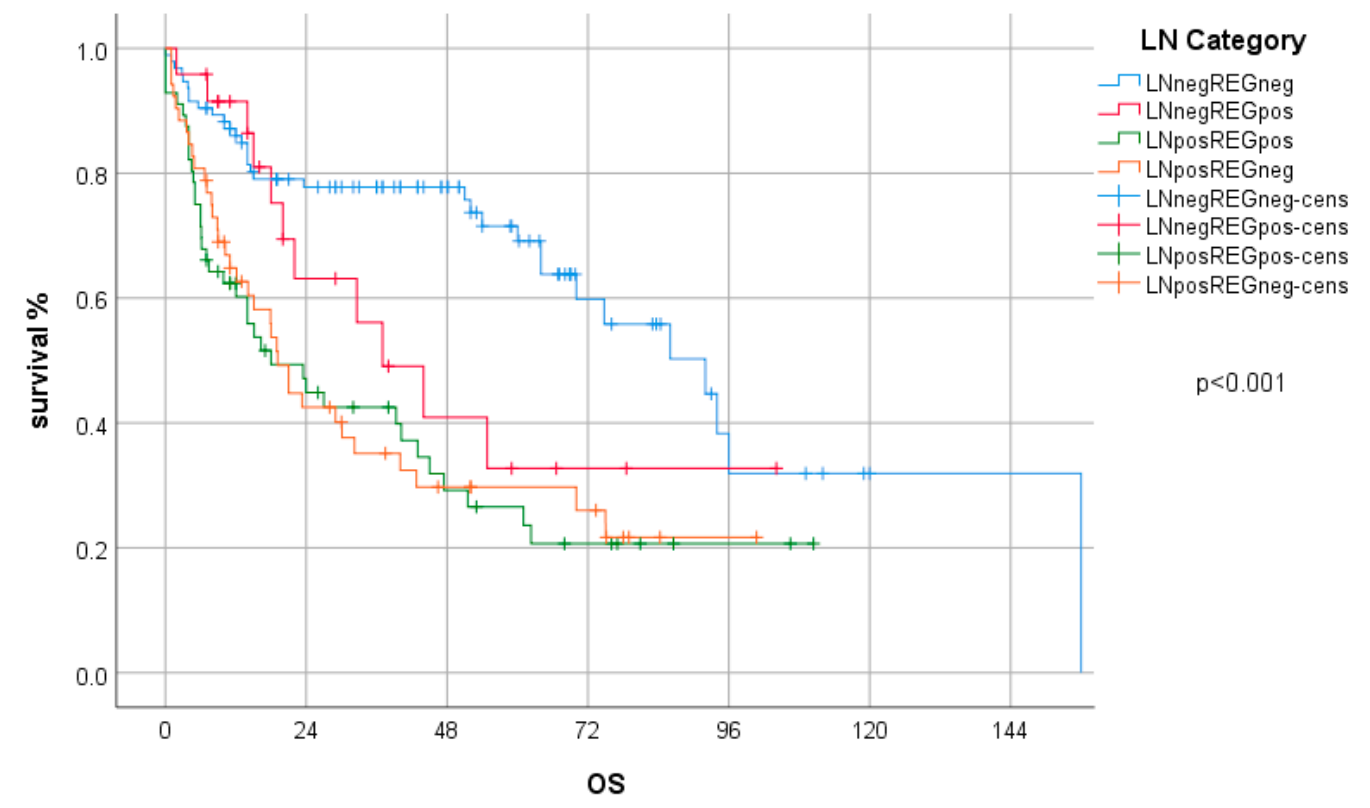

Figure 1. LN categories and overall survival (OS).

Other prognostically relevant factors in univariate analyses were TRG according to Becker, ypT-category, ypN-category, ypM-category (each $p<0.001$ ) and resection status $(p=0.031)$.

The LN/REG categorization was also an independent prognostic factor in a multivariate analysis $($ Hazard ratio $(\mathrm{HR})=1.32 ; 95 \%$ CI 1.11-1.58; $p=0.002)$, including TRG, ypT category, distant metastases and resection status (Table 3 ). The HR and significance levels for the LN/REG categorization were similar to those of the $\mathrm{ypN}$ categories according to the TNM classification, which also proved to be an independent prognostic parameter in multivariate analysis including the same influencing variables as mentioned above $(\mathrm{HR}=1.28 ; 95 \%$ CI 1.05-1.57; $p=0.016)$.

Table 3. Multivariate Analysis.

\begin{tabular}{ccccc}
\hline & HR & \multicolumn{2}{c}{ 95\% CI for HR } & $p$-Value \\
\hline & & Lower & Upper & \\
\hline ypTcategory & 1.118 & 0.874 & 1.430 & 0.376 \\
TRG (Becker) & 1.094 & 0.845 & 1.417 & 0.496 \\
LN category & 1.324 & 1.108 & 1.583 & 0.002 \\
distant metastases & 2.492 & 1.412 & 4.400 & 0.002 \\
resection status & 0.754 & 0.444 & 1.280 & 0.295 \\
\hline
\end{tabular}

\subsection{Subgroup Analysis-Case Collections and Type of Neoadjuvant Treatment}

The two case collections differed regarding the following parameters: in the Bern cohort, radiochemotherapy was the predominantly used neoadjuvant therapy regime, while in the Munich cohort only neoadjuvant chemotherapy was used $(p<0.001)$. Although the number of patients who achieved complete regression of the primary tumor was significantly higher in the Bern cohort $(p<0.001)$, the overall survival did not significantly differ $(p=0.537)$. This was also the case for the comparison between neoadjuvant RCTX and CTX, the latter group also including cases from Bern: neoadjuvant RCTX was associated with a higher rate of complete regression of the primary tumor $(p<0.001)$ but not with overall survival $(p=0.223)$. Regarding the LN/REG categories, in the Bern cohort and the RCTX group, the percentage of LN-/REG- and LN-/REG+ cases was higher compared 
to the Munich cohort and the CTX group, respectively $(p=0.003$; and $p=0.002)$. The overall prognostic impact for the LN/REG categorization was significant in all subgroups (Bern cohort: $p<0.001$; Munich cohort: $p=0.011$; RCTX group: $p=0.001$; CTX group: $p=0.003$ ), which was also seen in the multivariate analyses (Bern cohort: $\mathrm{HR}=1.19 ; 95 \% \mathrm{CI}=0.938-1.510 ; p=152$; Munich cohort: $\mathrm{HR}=1.484 ; 95 \% \mathrm{CI}=1.122-1.962 p=0.006 ; \mathrm{RCTX}$ group: $\mathrm{HR}=1.197 ; 95 \% \mathrm{CI}=0.912-1.570 p=0.195$; CTX group: $\mathrm{HR}=1.452 ; 95 \% \mathrm{CI}=1.132-1.862 ; p=0.003$; for details see Table 1 and supplemental data Figures S1-S4 and Tables S1-S4).

\section{Discussion}

We investigated the impact of regressive changes in lymph nodes (LN) or LN metastases (LNM), in particular, on survival in locally advanced esophageal adenocarcinomas (EAC) after neoadjuvant chemo- or radiochemotherapy (CTX or RCTX) using a dichotomous classification of two parameters, according to which the $\mathrm{LN}$ were categorized by presence or absence of residual metastases (LN- or $\mathrm{LN}+$ ) and regression characteristics (REG- or REG+).

We observed regressive changes in approximately 30\%-among them complete regression of previous metastases in occurred $10 \%$ of the total number of patients. On the other hand, there was total absence of regressive changes in LN metastases in $25 \%$ of the cases. We observed a significant positive correlation between the regression of the primary tumors and the LN metastases. Moreover, we demonstrated, that the group of patients with truly negative nodal status (LN-/REG-) as well as those with complete regression of previous LN metastases (LN-/REG+) had a better overall survival than those cases with residual metastases in the LN, regardless of whether with or without regressive changes (LN+/REG+ or LN+/REG-). However, occurrence of regressive changes in the subgroups of both ypN0 and ypN+ patients did not result in a significant survival benefit taking these groups alone. The prognosis of LN-/REG+ was worse than LN-/REG- but better than both LN+ categories, which was clearly seen in the Kaplan-Meier curves but did not reach statistical significance $(p=0.104$ and $p=0.090$, respectively). Therefore, the ypN status proved to be the most prognostically relevant parameter in our study.

According to previously published work, tumor shrinkage, fibrosis, acellular mucin, histiocytic reaction with foamy macrophages, calcification, necrotic areas have been suggested as main histopathological signs of regressive changes after neoadjuvant therapy $[23,24]$. The histopathological characteristics of regressive changes (e.g., fibrosis, necrosis, acellular mucin, histiocytic reactions and calcification) are the same for primary tumors and lymph nodes. We defined central fibrosis and acellular mucin as characteristic for regression. However, fibrosis cannot only be considered a therapeutic consequence of chemoradiotherapy but it can also be interpreted as a consequence of other non-specific inflammatory reactions [25-28]. Even in patients who have not received chemoradiotherapy, fibrosis can occur in the sense of a body's own defensive reaction to tumor cells. However, it has been demonstrated before that the interobserver agreement for the determination of regressive changes in LNM is within substantial levels [13,29].

In upper gastrointestinal cancers LN staging has a very high prognostic value [14]. For tumors treated with multimodal therapy, previous studies postulated that, not only the ypN status, but also the additional reporting and categorization of lymph node metastasis regression, may have important prognostic value $[13,23,29-34]$.

Different approaches on the classification of regressive changes have been described. Bollschweiler et al. [35] introduced a classification with three grades: low risk (no LN metastasis and fewer than three LNs with central fibrosis), medium risk (no LN metastasis and central fibrosis in three or more LNs, or LN metastasis with a LN ratio of less than 0.05 ) and high risk (all other cases). A previous study of our group on gastric cancer [29] proposed a dichotomous classification, which focused only on the assessment of whether regression was present in any of the investigated lymph nodes. This system had been proposed by an expert panel for the application in gastric cancer [22]. We therefore decided to apply this system on the esophageal adenocarcinoma cohort, also allowing a direct comparison to 
the cohort of gastric carcinomas reported previously. The frequency of regressive changes of about $30 \%$ in LN metastases of a patient in our study is comparable to other studies, both for esophageal and gastric cancer $[13,23,29,36]$. One study by Zhu et al. [37], however, reported a probability of occurrence of about $64 \%$, which is as twice as much compared to other studies. Similarities of this work with our study are the use of the same classification and the size of the patient population studied. Differences are the entity of the primary tumor (stomach vs. esophagus) and the ethnic background of the patients, which would explain this outstanding difference, compared to other studies.

The majority of studies on gastric and esophageal carcinomas agree on the prognostic significance of the presence or absence of LN metastases: Patients with ypN0, whether with or without regression in their LN metastases, have a clear survival advantage compared to patients with ypN+ [13,23,29,31,35,37,38].

Moreover, there are several studies that suggest that regression following neoadjuvant treatment may also have a prognostic value: in a subsequent work, Bollschweiler et al. were able to demonstrate that cases with metastasis-free LN and less than three regression features in terms of central fibrosis have a significantly better survival than those with more than three central fibrosis or even vital LN metastases [31,35]. They conclude that regressively altered LN seem to be former metastases and that, with respect to the LN- group, an increasing number of regressive lymph nodes additionally worsens survival. It is hypothesized that with increasing number of LN metastases, whether vital or regressive, the probability of micrometastases increases, which may explain the deterioration in overall survival. The fact, however, that this type of classification combines "true" ypN0 patients with "false" ypN0 patients (i.e., former N+ patients) in one tier impedes a clear differentiation regarding the prognosis of these two collectives. Likewise, studies in line with our classification did not achieve the establishment of significant prognostic differences between the cases with LN-/REG- and LN-/REG+. Nieman et al. [23] suggest that patients with former LN metastases with complete regression should be staged as ypN+, because LN with complete regression have a comparable negative influence on survival to positive LN. This, in turn, confirms the necessity of introducing a classification for lymph node staging that also takes regression into account.

In gastric cancer [29], but in contrast to this study on esophageal adenocarcinomas, we were able to demonstrate a significant prognostic advantage of the presence over absence of regressive changes in nodal positive patients. Since this was observed both in the RCTX and the CTX group, the tumor entity rather than the type of neoadjuvant treatment may be responsible for this difference.

The study has some limitations. First, it is a retrospective analysis and not associated to a clinical trial. Second, the cases collections generated from historical cohorts and novel treatment approaches may have a different effect on regression of the primary tumors and metastases. Third, we did not record the number of affected or regressed LNM, respectively. According to some studies, this factor plays an important role in estimating the prognosis of a patient, especially in ypN0 cases. In our study, however, a classification was used that does not consider the number of regressive changes-this may be much more practical in terms of cost and benefit and clinical consequence, which becomes increasingly important in medicine.

Lastly, the number of patients with former, post-therapeutically, completely regressed LN was rather low. This may explain the lack of significance levels in the comparison to the LN-/REG- and $\mathrm{LN}+$ groups despite a clear visible trend in the Kaplan-Meier curves. Since these trends go in line with the results of other studies, we conclude that the detection of a former LNM separates a patient from those who had always tumor free LN and that this warrants the report of regressive changes or the description of a completely regressed LNM in pathological reports. Interestingly, in the group of LN+ patients however, our results do not show a survival benefit of regression when there is still residual tumor detectable.

In summary, our data show that tumor regression of LN metastases can be observed in a substantial number of EAC after neoadjuvant therapy. The most relevant prognostic impact may be observed in the group of patients with former LN metastases that have completely regressed but should not be staged as simple ypN0. Reporting of regressive changes in LN, or LN metastases, respectively, 
should be integrated in pathology reports of upper gastrointestinal cancers which is already performed by a substantial number of gastrointestinal pathologists according to a recent survey [39].

\section{Materials and Methods}

\subsection{Patients}

The study included a total of 239 patients with locally advanced adenocarcinoma of the esophagus (EAC) as defined by the WHO classification [40] who were treated with neoadjuvant chemotherapy or chemoradiotherapy. Both collectives have been described in detail before and differed in their therapeutic approach [41,42]: The first collective comprised 92 cases, treated between 1994 and 2002 in the department of surgery of the Technische Universität München, Munich [42]. Neoadjuvant treatment consisted of a cisplatin/5-fluorouracil/Leucovorine-based chemotherapy with or without additional paclitaxel. Surgical resection of the tumors was performed by transmediastinal or transthoracal (Ivor Lewis) esophagectomy.

The second collective comprised 147 patients, treated between 2001 and 2016 at the department of Surgery of the Inselspital University Hospital Bern [41]. Neoadjuvant treatment was predominantly composed of combined radiation with at least 40 gray and a 5-fluorouracil and Cisplatin/Carboplatin-based chemotherapy with or without additional Paclitaxel or Docetaxel. Neoadjuvant treatment was followed by transmediastinal esophageal resection with a radical bilateral mediastinal en-bloc lymphadenectomy.

Patient data were taken from prospectively managed databases. Follow-up included endoscopy and CT scans at intervals of 6 to 12 months over at least 5 years according to institutional protocols. Survival was computed from the day of surgery. More details of the patient groups can be found in Table 1. The usage of pathologic and clinical data for research had been approved by the ethics committees of the Medical Faculty of the Technische Universität München, 2056/08 and the Canton of Bern, 2017-01623.

\subsection{Post Neoadjuvant TNM Staging and Tumor Regression Grading}

Histopathological examination of the resection specimens and the assessment of TNM categories was performed as described before [41-43]. Data for histopathological tumor regression according to Becker (Tumor regression grade (TRG) $1 \mathrm{a}=$ complete regression; TRG1b $=<10 \%$ residual tumor; TRG2 $=10-50 \%$ residual tumor; TRG3 $=>50 \%$ residual tumor) and determined by experienced pathologists with a special focus on gastrointestinal tumors (KB, RL) were obtained from previous studies $[41,42]$ or generated for the purpose of the present work.

\subsection{Evaluation of Tumor Regression in Lymph Node Metastases}

The lymph nodes (LN) of all 239 cases were examined for histopathological features of regression by three observers (KB, RL, MO). The presence of hyaline fibrosis, acellular mucin or sheets of foamy histiocytes were defined as characteristic for therapy induced regression (Figure 2), in line with previous reports $[23,24,29]$. We then applied the categorization system, which was already described for gastric cancer [22,37]: it distinguishes between the presence and absence of regression (REG- or $\mathrm{REG}+$ ) in $\mathrm{LN}$ along with the absence or presence of residual tumor ( $\mathrm{LN}-$ or $\mathrm{LN}+$ ), regardless of the number of affected LN and without further graduation regarding the extent of regression or number of regressed LNM $[13,31]$. By combining these items, the following categories can be defined: LN-/REG-: ypN0 category without signs of regression; LN-/REG+: ypN0 category with evidence of regression; LN+/REG+: ypN1-3 categories with evidence of regression; LN+/REG-: ypN1-3 categories without signs of regression. 

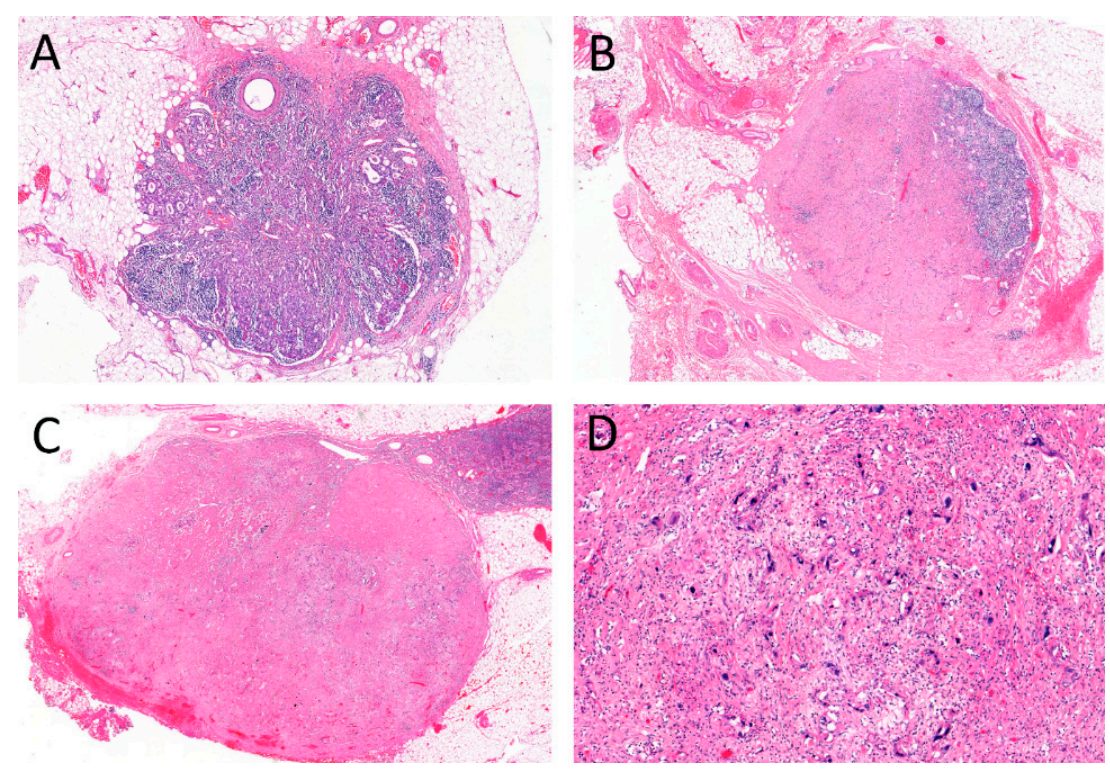

Figure 2. Examples of lymph node metastases (LNM): (A) LNM without regression (LN+/REG-); (B) LN with completely regressed former metastasis (LN-/REG+); (C) LNM with regression and residual tumor $(\mathrm{LN}+/ \mathrm{REG}+)$; higher magnification of $(\mathrm{C})$; all images magnification $\times 10$, except $(\mathbf{D})(\times 40)$.

\subsection{Statistical Analysis}

The statistical analyses were carried out using IBM SPPS Statistics 26 (IBM Corporation, Armonk, NY, USA). Comparisons between groups and categories were made performed using cross tables, $\chi^{2}$ tests, and Fisher's exact tests. Survival analysis included Kaplan-Meier curves and log-rank tests for univariate survival analysis and Cox regression analysis (enter method) for multivariate analysis. $p$-values of $<0.05$ were considered significant for all statistical tests.

Supplementary Materials: The following are available online at http://www.mdpi.com/2624-5647/2/4/36/s1, Figure S1: Bern cohort: LN categories and overall survival Table S1: Bern cohort: multivariate analysis, Figure S2 Munich cohort: LN categories and overall survival, Table S2: Munich cohort: multivariate analysis, Figure S3: RCTX patients and overall survival, Table S3: RCTX patients: multivariate analysis, Figure S4: CTX patients and overall survival, Table S4: CTX patients: multivariate analysis.

Author Contributions: Conceptualization, M.O. and R.L.; validation, K.B.; investigation, M.O., R.L., K.B.; data curation, M.O., D.K., M.F., D.R., B.D., K.B., R.L.; writing一original draft preparation, M.O. and R.L.; writing一review and editing R.L., D.R. visualization, M.O., R.L.; All authors have read and agreed to the published version of the manuscript.

Funding: This research received no external funding.

Conflicts of Interest: The authors declare no conflict of interest.

\section{References}

1. Allum, W.H.; Stenning, S.P.; Bancewicz, J.; Clark, P.I.; Langley, R.E. Long-Term Results of a Randomized Trial of Surgery With or Without Preoperative Chemotherapy in Esophageal Cancer. J. Clin. Oncol. 2009, 27, 5062-5067. [CrossRef] [PubMed]

2. Medical Research Council Oesophageal Cancer Working Group. Surgical resection with or without preoperative chemotherapy in oesophageal cancer: A randomised controlled trial. Lancet 2002, 359, 1727-1733. [CrossRef]

3. van Hagen, P.; Hulshof, M.C.C.M.; van Lanschot, J.J.B.; Steyerberg, E.W.; van Berge Henegouwen, M.I.; Wijnhoven, B.P.L.; Richel, D.J.; Nieuwenhuijzen, G.A.P.; Hospers, G.A.P.; Bonenkamp, J.J.; et al. Preoperative Chemoradiotherapy for Esophageal or Junctional Cancer. N. Engl. J. Med. 2012, 366, 2074-2084. [CrossRef] [PubMed] 
4. Cunningham, D.; Allum, W.H.; Stenning, S.P.; Thompson, J.N.; Van De Velde, C.J.; Nicolson, M.; Scarffe, J.H.; Lofts, F.J.; Falk, S.J.; Iveson, T.J.; et al. Perioperative Chemotherapy versus Surgery Alone for Resectable Gastroesophageal Cancer. N. Engl. J. Med. 2006, 355, 11-20. [CrossRef]

5. Pasquali, S.; Yim, G.; Vohra, R.S.; Mocellin, S.; Nyanhongo, G.S.; Marriott, P.; Geh, J.I.; Griffiths, E.A. Survival After Neoadjuvant and Adjuvant Treatments Compared to Surgery Alone for Resectable Esophageal Carcinoma. Ann. Surg. 2017, 265, 481-491. [CrossRef]

6. Ronellenfitsch, U.; Schwarzbach, M.; Hofheinz, R.; Kienle, P.; Kieser, M.; Slanger, T.E.; Jensen, K. GE Adenocarcinoma meta-Analysis Group. Perioperative chemo(radio)therapy versus primary surgery for resectable adenocarcinoma of the stomach, gastroesophageal junction, and lower esophagus. Cochrane Database Syst. Rev. 2013. [CrossRef]

7. Gebski, V.; Burmeister, B.; Smithers, B.M.; Foo, K.; Zalcberg, J.; Simes, J. Survival benefits from neoadjuvant chemoradiotherapy or chemotherapy in oesophageal carcinoma: A meta-analysis. Lancet Oncol. 2007, 8, 226-234. [CrossRef]

8. Sjoquist, K.M.; Burmeister, B.H.; Smithers, B.M.; Zalcberg, J.R.; Simes, R.J.; Barbour, A.; Gebski, V. Survival after neoadjuvant chemotherapy or chemoradiotherapy for resectable oesophageal carcinoma: An updated meta-analysis. Lancet Oncol. 2011, 12, 681-692. [CrossRef]

9. Klevebro, F.; Johnsen, G.; Johnson, E.; Viste, A.; Myrnäs, T.; Szabo, E.; Jacobsen, A.-B.; Friesland, S.; Tsai, J.; Persson, S.; et al. Morbidity and mortality after surgery for cancer of the oesophagus and gastro-oesophageal junction: A randomized clinical trial of neoadjuvant chemotherapy vs. neoadjuvant chemoradiation. Eur. J. Surg. Oncol. (EJSO) 2015, 41, 920-926. [CrossRef]

10. Klevebro, F.; Von Döbeln, G.A.; Wang, N.; Johnsen, G.; Jacobsen, A.-B.; Friesland, S.; Hatlevoll, I.; Glenjen, N.I.; Lind, P.; Tsai, J.A.; et al. A randomized clinical trial of neoadjuvant chemotherapy versus neoadjuvant chemoradiotherapy for cancer of the oesophagus or gastro-oesophageal junction. Ann. Oncol. 2016, 27, 660-667. [CrossRef]

11. Mandard, A.M.; Dalibard, F.; Mandard, J.-C.; Marnay, J.; Henry-Amar, M.; Petiot, J.-F.; Roussel, A.; Jacob, J.-H.; Segol, P.; Samama, G.; et al. Pathologic assessment of tumor regression after preoperative chemoradiotherapy of esophageal carcinoma. Clinicopathologic correlations. Cancer 1994, 73, 2680-2686. [CrossRef]

12. Becker, K.; Mueller, J.; Schulmacher, C.; Ott, K.; Fink, U.; Busch, R.; Böttcher, K.; Siewert, J.R.; Höfler, H. Histomorphology and grading of regression in gastric carcinoma treated with neoadjuvant chemotherapy. Cancer 2003, 98, 1521-1530. [CrossRef]

13. Davies, A.; Myoteri, D.; Zylstra, J.; Baker, C.R.; Wulaningsih, W.; Van Hemelrijck, M.; Maisey, N.; Allum, W.H.; Smyth, E.; A Gossage, J.; et al. Lymph node regression and survival following neoadjuvant chemotherapy in oesophageal adenocarcinoma. Br. J. Surg. 2018, 105, 1639-1649. [CrossRef] [PubMed]

14. Becker, K.; Langer, R.; Reim, D.; Novotny, A.; Buschenfelde, C.M.Z.; Engel, J.; Friess, H.; Hofler, H. Significance of Histopathological Tumor Regression After Neoadjuvant Chemotherapy in Gastric Adenocarcinomas. Ann. Surg. 2011, 253, 934-939. [CrossRef] [PubMed]

15. Smyth, E.C.; Fassan, M.; Cunningham, D.; Allum, W.H.; Okines, A.F.; Lampis, A.; Hahne, J.C.; Rugge, M.; Peckitt, C.; Nankivell, M.; et al. Effect of Pathologic Tumor Response and Nodal Status on Survival in the Medical Research Council Adjuvant Gastric Infusional Chemotherapy Trial. J. Clin. Oncol. 2016, 34, 2721-2727. [CrossRef] [PubMed]

16. Talsma, A.; Ong, C.-A.J.; Liu, X.; Van Hagen, P.; Van Lanschot, J.J.B.; Tilanus, H.W.; Hardwick, R.H.; Carroll, N.R.; Spaander, M.C.W.; Fitzgerald, R.C.; et al. Location of Lymph Node Involvement in Patients with Esophageal Adenocarcinoma Predicts Survival. World J. Surg. 2013, 38, 106-113. [CrossRef] [PubMed]

17. Leers, J.M.; Demeester, S.R.; Chan, N.; Ayazi, S.; Oezcelik, A.; Abate, E.; Banki, F.; Lipham, J.C.; Hagen, J.A.; Demeester, T.R. Clinical characteristics, biologic behavior, and survival after esophagectomy are similar for adenocarcinoma of the gastroesophageal junction and the distal esophagus. J. Thorac. Cardiovasc. Surg. 2009, 138, 594-602. [CrossRef] [PubMed]

18. Barbour, A.; Jones, M.; Gonen, M.; Gotley, D.C.; Thomas, J.; Thomson, D.B.; Burmeister, B.; Smithers, B.M. Refining Esophageal Cancer Staging After Neoadjuvant Therapy: Importance of Treatment Response. Ann. Surg. Oncol. 2008, 15, 2894-2902. [CrossRef]

19. Hoelscher, A.; Bollschweiler, E.; Bogoevski, D.; Schmidt, H.; Semrau, R.; Izbicki, J. Prognostic impact of neoadjuvant chemoradiation in CT3 oesophageal cancer-A propensity score matched analysis. Eur. J. Cancer 2014, 50, 2950-2957. [CrossRef] 
20. Bollschweiler, E.; Metzger, R.; Drebber, U.; Baldus, S.; Vallböhmer, D.; Kocher, M.; Hölscher, A.H. Histological type of esophageal cancer might affect response to neo-adjuvant radiochemotherapy and subsequent prognosis. Ann. Oncol. 2009, 20, 231-238. [CrossRef]

21. Meredith, K.L.; Weber, J.M.; Turaga, K.K.; Siegel, E.M.; McLoughlin, J.; Hoffe, S.; Marcovalerio, M.; Shah, N.; Kelley, S.; Karl, R. Pathologic Response after Neoadjuvant Therapy is the Major Determinant of Survival in Patients with Esophageal Cancer. Ann. Surg. Oncol. 2010, 17, 1159-1167. [CrossRef]

22. Tsekrekos, A.; Detlefsen, S.; Riddell, R.; Conner, J.; Mastracci, L.; Sheahan, K.; Shetye, J.; Lundell, L.; Vieth, M. Histopathologic tumor regression grading in patients with gastric carcinoma submitted to neoadjuvant treatment: Results of a Delphi survey. Hum. Pathol. 2019, 84, 26-34. [CrossRef] [PubMed]

23. Nieman, D.R.; Peyre, C.G.; Watson, T.J.; Cao, W.; Lunt, M.D.; Lada, M.J.; Han, M.S.; Jones, C.E.; Peters, J.H. Neoadjuvant Treatment Response in Negative Nodes Is an Important Prognosticator After Esophagectomy. Ann. Thorac. Surg. 2015, 99, 277-283. [CrossRef] [PubMed]

24. Langer, R.; Becker, K. Tumor regression grading of gastrointestinal cancers after neoadjuvant therapy. Virchows Arch. 2018, 472, 175-186. [CrossRef] [PubMed]

25. Ott, K.; Weber, W.A.; Lordick, F.; Becker, K.; Busch, R.; Herrmann, K.; Wieder, H.; Fink, U.; Schwaiger, M.; Siewert, J.-R. Metabolic Imaging Predicts Response, Survival, and Recurrence in Adenocarcinomas of the Esophagogastric Junction. J. Clin. Oncol. 2006, 24, 4692-4698. [CrossRef] [PubMed]

26. Van Heijl, M.; Omloo, J.M.; Henegouwen, M.I.V.B.; Hoekstra, O.S.; Boellaard, R.; Bossuyt, P.M.; Busch, O.; Tilanus, H.W.; Hulshof, M.C.; Van Der Gaast, A.; et al. Fluorodeoxyglucose Positron Emission Tomography for Evaluating Early Response During Neoadjuvant Chemoradiotherapy in Patients With Potentially Curable Esophageal Cancer. Ann. Surg. 2011, 253, 56-63. [CrossRef]

27. Omloo, J.M.T.; Van Heijl, M.; Hoekstra, O.S.; Henegouwen, M.I.V.B.; Van Lanschot, J.J.B.; Sloof, G.W. FDG-PET parameters as prognostic factor in esophageal cancer patients: A review. Ann. Surg. Oncol. 2011, 18, 3338-3352. [CrossRef]

28. Piessen, G.; Petyt, G.; Duhamel, A.; Mirabel, X.; Huglo, D.; Mariette, C. Ineffectiveness of 18F-Fluorodeoxyglucose Positron Emission Tomography in the Evaluation of Tumor Response After Completion of Neoadjuvant Chemoradiation in Esophageal Cancer. Ann. Surg. 2013, 258, 66-76. [CrossRef]

29. Reim, D.; Novotny, A.; Friess, H.; Slotta-Huspenina, J.; Weichert, W.; Ott, K.; Dislich, B.; Lorenzen, S.; Becker, K.; Langer, R. Significance of tumour regression in lymph node metastases of gastric and gastro-oesophageal junction adenocarcinomas. J. Pathol. Clin. Res. 2020. [CrossRef]

30. Fernández-Aceñero, M.J.; Granja, M.; Sastre, J.; García-Paredes, B.; Estrada, L. Prognostic significance of tumor regression in lymph nodes after neoadjuvant therapy for rectal carcinoma. Virchows Arch. 2016, 468, 425-430. [CrossRef]

31. Philippron, A.; Bollschweiler, E.; Kunikata, A.; Plum, P.; Schmidt, C.; Favi, F.; Drebber, U.; Hölscher, A.H. Prognostic Relevance of Lymph Node Regression After Neoadjuvant Chemoradiation for Esophageal Cancer. Semin. Thorac. Cardiovasc. Surg. 2016, 28, 549-558. [CrossRef] [PubMed]

32. Kim, S.H.; Chang, H.J.; Kim, D.Y.; Park, J.W.; Baek, J.Y.; Kim, S.Y.; Park, S.C.; Oh,J.H.; Yu, A.; Nam, B.-H. What Is the Ideal Tumor Regression Grading System in Rectal Cancer Patients after Preoperative Chemoradiotherapy? Cancer Res. Treat. 2016, 48, 998-1009. [CrossRef] [PubMed]

33. Sannier, A.; Lefevre, J.H.; Panis, Y.; Cazals-Hatem, D.; Bedossa, P.; Guedj, N. Pathological prognostic factors in locally advanced rectal carcinoma after neoadjuvant radiochemotherapy: Analysis of 113 cases. Histopathology 2014, 65, 623-630. [CrossRef] [PubMed]

34. Noble, F.; Lloyd, M.A.; Turkington, R.; Griffiths, E.; O’Donovan, M.; O’Neill, J.R.; Mercer, S.; Parsons, S.L.; Fitzgerald, R.C.; Underwood, T.J.; et al. Multicentre cohort study to define and validate pathological assessment of response to neoadjuvant therapy in oesophagogastric adenocarcinoma. Br. J. Surg. 2017, 104, 1816-1828. [CrossRef] [PubMed]

35. Bollschweiler, E.; Hölscher, A.H.; Metzger, R.; Besch, S.; Moenig, S.P.; E Baldus, S.; Drebber, U. Prognostic Significance of a New Grading System of Lymph Node Morphology After Neoadjuvant Radiochemotherapy for Esophageal Cancer. Ann. Thorac. Surg. 2011, 92, 2020-2027. [CrossRef]

36. Donohoe, C.L.; O'farrell, N.J.; Grant, T.; King, S.; Clarke, L.; Muldoon, C.; Reynolds, J.V. Classification of Pathologic Response to Neoadjuvant Therapy in Esophageal and Junctional Cancer. Ann. Surg. 2013, 258, 784-792. [CrossRef] 
37. Zhu, Y.-L.; Sun, Y.-K.; Xue, X.-M.; Yue, J.-Y.; Yang, L.; Xue, L. Unnecessity of lymph node regression evaluation for predicting gastric adenocarcinoma outcome after neoadjuvant chemotherapy. World J. Gastrointest. Oncol. 2019, 11, 48-58. [CrossRef]

38. Martin-Romano, P.; Sola, J.J.; A Diaz-Gonzalez, J.; Chopitea, A.; Iragorri, Y.; Martínez-Regueira, F.; Ponz-Sarvise, M.; Arbea, L.; Subtil, J.C.; Cano, D.; et al. Role of histological regression grade after two neoadjuvant approaches with or without radiotherapy in locally advanced gastric cancer. Br. J. Cancer 2016, 115, 655-663. [CrossRef]

39. Westerhoff, M.; Osecky, M.; Langer, R. Varying practices in tumor regression grading of gastrointestinal carcinomas after neoadjuvant therapy: Results of an international survey. Mod. Pathol. 2019, 33, 676-689. [CrossRef]

40. Digestive System Tumours. WHO Classification of Tumours Editorial Board, 5th ed.; WHO: Geneva, Switzerland, 2019; Volume 1.

41. Kröll, D.; Noser, L.; Erdem, S.; Storni, F.; Arnold, D.; Dislich, B.; Zlobec, I.; Candinas, D.; Seiler, C.A.; Langer, R.; et al. Application of the 8th edition of the AJCC yTNM staging system shows improved prognostication in a single center cohort of esophageal carcinomas. Surg. Oncol. 2018, 27, 100-105. [CrossRef]

42. Langer, R.; Ott, K.; Feith, M.; Lordick, F.; Siewert, J.-R.; Becker, K. Prognostic significance of histopathological tumor regression after neoadjuvant chemotherapy in esophageal adenocarcinomas. Mod. Pathol. 2009, 22, 1555-1563. [CrossRef] [PubMed]

43. Brierley, J.; Gospodarowicz, M.K.; Wittekind, C. (Eds.) TNM Classification of Malignant Tumours, 8th ed.; Wiley: Hoboken, NI, USA, 2017.

Publisher's Note: MDPI stays neutral with regard to jurisdictional claims in published maps and institutional affiliations.

(C) 2020 by the authors. Licensee MDPI, Basel, Switzerland. This article is an open access article distributed under the terms and conditions of the Creative Commons Attribution (CC BY) license (http://creativecommons.org/licenses/by/4.0/). 